\title{
ANÁLISIS DEL COMPORTAMIENTO DEL ROBOT HUMANOIDE BIOLOID EN SITUACIÓN ESPECÍFICA
}

\author{
BEHAVIORAL ANALYSIS OF HUMANOID ROBOT BIOLOID IN SPECIFIC SITUATION
}

\author{
Jonathan Alexander Soto- \\ Montoya, Est. \\ Fundación Universitaria Luis Amigó \\ Grupo de investigación SISCO. \\ Medellín, Colombia \\ jonathan.sotomo@amigo.edu.co
}

\author{
Julio Cesar Gómez-Naranjo, \\ Est. \\ Fundación Universitaria Luis Amigó \\ Grupo de investigación SISCO. \\ Medellín, Colombia \\ julio.gomezna@amigo.edu.co
}

\author{
Jaime Alberto Guzmán- \\ Luna, Ph.D \\ Universidad Nacional de Colombia \\ Facultad de Minas \\ Grupo de investigación SINTELWEB \\ Medellín, Colombia \\ jaguzman@unal.edu.co
}

Resumen. Inicialmente, este trabajo aborda un problema que consiste en buscar un método de solución a una situación simulada de desbordamiento de un río, y su respectiva solución de manera autónoma por parte de un robot humanoide (en este caso un robot Bioloid); pues los de este tipo se desempeñan mejor que los robots Rover en terrenos ásperos al usar piernas en lugar de ruedas. Posteriormente se analizan los métodos realizados por el robot para la consecución de su objetivo y cómo es el desempeño de dichos métodos en índoles importantes en este tipo de situaciones; por ejemplo, aspectos como su velocidad, el tiempo de ejecución de la situación y su grado de error.

Palabras clave: Autonomía, Bioloid, humanoide, robótica, simulación de catástrofe.

\begin{abstract}
Initially, this text tackles a problem that consists in looking for a solution way about a simulated overflow river, and its autonomously solution by a humanoid robot (in this case, the robot used is a Bioloid); because this kind of robots are better than Rover robots over rough terrain as it uses legs instead of wheels.

Later, this text analyzes the ways taken by the robot to reach its goal and the performance of such ways from different topics, like speed, execution time of the situation and its degree of error.
\end{abstract}

Keywords: Autonomy, Bioloid, humanoid, robotics, disaster simulation. 


\section{INTRODUCCIÓN}

La robótica es una rama de la tecnología que ha tomado un gran auge en los últimos tiempos y que a lo largo de su historia [1] ha ayudado a la humanidad en áreas como la salud, la domótica, la industria textil, la industria automovilística, entre otras; sin embargo, esta rama de la tecnología no ha sido lo suficientemente dedicada a la atención de desastres, y teniendo en cuenta que se podrían salvar muchas vidas, se hace pertinente abordar la robótica con orientación a este tema. También se hace pertinente analizar uno de sus problemas fundamentales como lo es la llamada incertidumbre, la cual básicamente trata del grado de error que los dispositivos robóticos -o cualquier partícula- pueden generar [2]. Esta incertidumbre será evaluada en una serie de pruebas, como lo son caminar, girar cierta cantidad de grados, coger o dejar un pilote; con el objetivo de posteriormente conocer y puntualizar exactamente qué tanto afecta la incertidumbre los movimientos a largo plazo.

Actualmente, como ya se mencionó, las investigaciones de robótica de esta índole son escasas; pues estas se concentran más en el sector productivo. Aunque proporcionalmente hablando el sector de atención y prevención de desastres es variado en cuanto a prototipos robóticos se refiere; empezando con los robots CA-TF2 y VA-TF1 [3], los cuales proporcionan una gran ayuda humanitaria en condiciones adversas.

Después de un terremoto o alguna catástrofe es bien sabido el daño emocional en las personas, por lo que las investigaciones en robótica también se centran en ayudar anímicamente a las personas; por ejemplo, Paro [4], un robot autómata [5] que se convierte en un claro ejemplar de ayuda emocional a las personas. En las inundaciones también se pueden encontrar robots que salvan vidas como es el caso Swumanoid [6], el cual rescata personas de dichas situaciones llegando hasta ellas nadando.

Por ello en este artículo se abordará el aspecto de las inundaciones, con el modelo robótico Bioloid en la situación específica de atención de un río desbordado, el cual también tiene que darse a la tarea de hacerlo eficazmente, ya que de la eficiencia del robot dependerá la correcta atención de la situación.

\section{DESARROLLO DEL ARTíCULO}

\subsection{Descripción del problema}

El problema básicamente se concibió con el fin de probar el desempeño del conjunto de los algoritmos desarrollados en orientación a los movimientos básicos y probar cómo es su funcionamiento individual y grupal. Dicho problema consiste en la simulación de un desbordamiento de un río, el cual debe ser solucionado por el robot de manera que ponga una cantidad determinada de pilotes alrededor del desbordamiento a fin de construir un dique que lo contenga. Además de esto se debe buscar que el robot haga esta tarea lo más eficaz y eficientemente posible para reducir los daños que provoque el ya mencionado desbordamiento.

\subsubsection{Campo}

El campo que se utilizó; como lo muestra la Figura 1, consta de una columna de color azul al lado izquierdo en representación de un río, dentro de las condiciones del problema se utilizó una serie de cuadros o cajas de color azul claro al lado derecho del río, las cuales tuvieron la función de simular el desborde; la posición de estos es aleatoria a fin de hacer la tarea más real. En cuanto a los pilotes; representados con el color verde, se ubican de forma constante en el extremo derecho del campo y 2 más en la parte superior e inferior en posición perpendicular respecto a los demás. La cuadrícula de color amarillo representa la posición inicial del robot; para la ubicación del robot en la pista se ha hecho un sistema de coordenadas como es mostrado en la Figura 1.

\subsubsection{Pilotes}

El campo también contendrá una serie de pilotes ubicados al extremo derecho de la pista como se ve en la Figura 1; estos serán usados por el robot para cubrir el desborde del río simulando la construcción de un dique. Estos pilotes están hechos por trozos de tubos PVC (policloruro de vinilo) con trozos de cartón adheridos en la parte de arriba para que el robot tenga más posibilidades de cogerlo.

\subsubsection{Robot}

El robot que se va a usar será el Bioloid Premium, distribuido por la empresa Robotis [7]; este, se ha armado con el modelo Tipo A [8] con 2 modificaciones 


\begin{tabular}{|r|r|r|r|r|r|}
\hline 0,0 & 0,1 & 0,2 & 0,3 & 0,4 & 0,5 \\
\hline 1,0 & 1,1 & 1,2 & 1,3 & 1,4 & 1,5 \\
\hline 2,0 & 2,1 & 2,2 & 2,3 & 2,4 & 2,5 \\
\hline 3,0 & 3,1 & 3,2 & 3,3 & 3,4 & 3,5 \\
\hline 4,0 & 3,2 & 4,2 & 4,3 & 4,4 & 4,5 \\
\hline 5,0 & 3,3 & 5,2 & 5,3 & 5,4 & 5,5 \\
\hline 6,0 & 6,1 & 6,2 & 6,3 & 6,4 & 6,5 \\
\hline 7,0 & 7,1 & 7,2 & 7,3 & 7,4 & 7,5 \\
\hline 8,0 & 8,1 & 8,2 & 8,3 & 8,4 & 8,5 \\
\hline 9,0 & 9,1 & 9,2 & 9,3 & 9,4 & 9,5 \\
\hline
\end{tabular}

Figura 1. Campo utilizado para las pruebas.

claves; la primera, es que se le ha puesto un caucho a cada mano del robot para garantizar que pueda coger los pilotes; la segunda se trata de los sensores infrarrojos [9] que se le han adaptado a cada pie apuntando hacia el frente para que pueda "ver" si el desbordamiento está en frente.

\subsection{Arquitectura para la solución}

Para la solución del caso del desbordamiento propuesto, se implementó una arquitectura [10] de búsqueda y solución; la cual, se concibió grosso modo un pseudocódigo que fue la base para programar el robot Bioloid para que con su implementación, cumpla con los objetivos y posteriormente permitan el análisis de los mismos. Las tareas principales que cumple el robot son:

- Caminar()

- GirarDerecha()

- Girarlzquierda()

- SensarAdelante()

- CogerPilote()

- DejarPilote()

Teniendo ya estas acciones básicas elaboradas, se construyó el pseudocódigo y posteriormente se implementó en el RoboPlus Task [11] y Roboplus Motion [12]:
Inicio

Caminar(2)

orientacion $=3$

fila $=1$

columna $=4$

contadorDesborde $=1$

Repetir hasta que contador_desborde sea diferente de 4

Si hay un obstáculo en frente Ir_Por_Pilote()

Sino

Girarlzquierda()

Caminar(1)

GirarDerecha()

Fin_si

Fin

Fin_repetir

El método Ir_Por_Pilote() es utilizado cuando el robot encuentra un desborde y lo que hace es evaluar la posición en la que actualmente se encuentra el robot y definir el camino óptimo para ir por los pilotes más cercanos y usarlos para hacer el dique.

Inicio

Si ContadorDesborde es igual a 1

Si la posición actual es 1,2

Ir por el pilote de la posición 0,3 y ponerlo en la posición actual

Ir por el pilote de la posición 0,4 y ponerlo en la posición 0,1

Ir por el pilote de la posición 0,5 y ponerlo en la posición 0,2

Sino, si la posición actual es 2,2

Ir por el pilote de atrás (posición 2,5) y dejarlo en la posición actual

Ir por el pilote de la posición 1,4 y ponerlo en la posición 1,1

Ir por el pilote de la posición 0,3 y ponerlo en la posición 1,2

Sino

Ir por el pilote de atrás $(x, 5)$ y ponerlo en la posición actual $(\mathrm{a}, \mathrm{b})$

Ir por el pilote de la posición $(x-1,5)$ y ponerlo en la posición (a-1, b-1)

Ir por el pilote de la posición $(x-2,5)$ y ponerlo en la posición $(a-1, b)$

Fin_si

Sino, si contadorDesborde es igual a 2

Ir por el pilote de atrás y ponerlo en la posición actual

Girarlzquierda () 
Si la posición actual es igual a 8,3

CogerPilote ()

Caminar (1)

GirarDerecha ()

DejarPilote ()

Sino

Caminar (1)

GirarDerecha ()

Caminar (1)

Sino

Fin_si

Si la posición actual es 8,2

Ir por el pilote de la posición 9,3 y ponerlo

en la posición actual

Ir por el pilote de la posición 9,4 y ponerlo en la posición 9,1

Ir por el pilote de atrás (posición 9,5) y dejarlo en la posición actual

Sino

Ir por el pilote de atrás $(x, 5)$ y dejarlo en la posición actual $(a, b)$

Ir por el pilote de la posición $x+1,5$ y ponerlo en la posición $a+1, b-1$

GirarDerecha ()

GirarDerecha ()

Caminar (1)

Si la posición actual es 8,3

GirarDerecha ()

CogerPilote ()

GirarDerecha ()

Sino

Caminar (2)

GirarDerecha ()

CogerPilote ()

GirarDerecha ()

Caminar (2)

Fin_si

Fin_si

DejarPilote ()

Fin_si Fin

contadorDesborde $=$ contadorDesborde +1

Para que el robot sepa en qué lugar se encuentra y pueda evaluar estas condiciones se ha concebido un sistema que suma o resta a la variable fila o columna cuando camina hacia adelante según su orientación; y esta a su vez es modificada cada vez que el robot gira.

\subsection{Pruebas y resultados}

Después de definido el algoritmo para cada uno de los movimientos básicos, se tomaron individualmente para probar el desempeño de estos en su ejecución; primero, se realizaron pruebas al movimiento caminar; cada una de ellas se hizo con diferentes distancias a fin de determinar la velocidad y los grados de error que arroja cada una; los resultados de aquella están expresados en la Tabla 1 y los de estos en la Figura 2.

Después, al igual que a caminar, se realizó varias pruebas al movimiento básico de girar; cada una de ellas se hizo con diferentes grados objetivo a fin de determinar la velocidad y los grados de error que arroja cada una; los resultados de aquella se pueden ver en la Tabla 2 y los de estos en la Figura 3.

Gracias a los cauchos instalados en las manos del robot, y los cartones que se le adhirieron alrededor del pilote, los cuales tenían la función de ampliar el diámetro del pilote; el proceso de coger y dejar se hizo significativamente más eficaz, dando como resultados un $100 \%$ para las pruebas de coger el pilote y un $90 \%$ para las pruebas de dejar estando el pilote en una posición adecuada.

\section{TRABAJOS FUTUROS}

En trabajos posteriores se pretende buscar métodos para reducir el grado de incertidumbre que se presenta al realizar los movimiento básicos; y mejorar el tiempo en que ejecuta dichas acciones, de igual manera mejorar sus cualidades motrices [13].

También se pretende incursionar en otras situaciones de catástrofe; por ejemplo, incendios, inundaciones, terremotos, entre otros. Con el objetivo de demostrar cómo estos robots sirven de herramientas para situaciones determinadas.

\section{CONCLUSIONES}

Las pruebas revelaron la gran incertidumbre que se presenta en un robot humanoide; ya que como se puede ver en las figuras de las pruebas, en unas secciones se presenta en gran grado la incertidumbre; y en otras, es verdaderamente mínima; demostrando con esto que la robótica depende mucho más del hardware [14] que del software [15]. 
TABLA 1

RESULTADOS DE VELOCIDAD CUANDO EL ROBOT CAMINA

\begin{tabular}{cc}
\hline Distancia & Velocidad promedio \\
\hline $20 \mathrm{~cm}$ & $0,66 \mathrm{~cm} / \mathrm{s}$ \\
$40 \mathrm{~cm}$ & $0,64 \mathrm{~cm} / \mathrm{s}$ \\
$60 \mathrm{~cm}$ & $0,43 \mathrm{~cm} / \mathrm{s}$ \\
$80 \mathrm{~cm}$ & $0,58 \mathrm{~cm} / \mathrm{s}$ \\
\hline
\end{tabular}

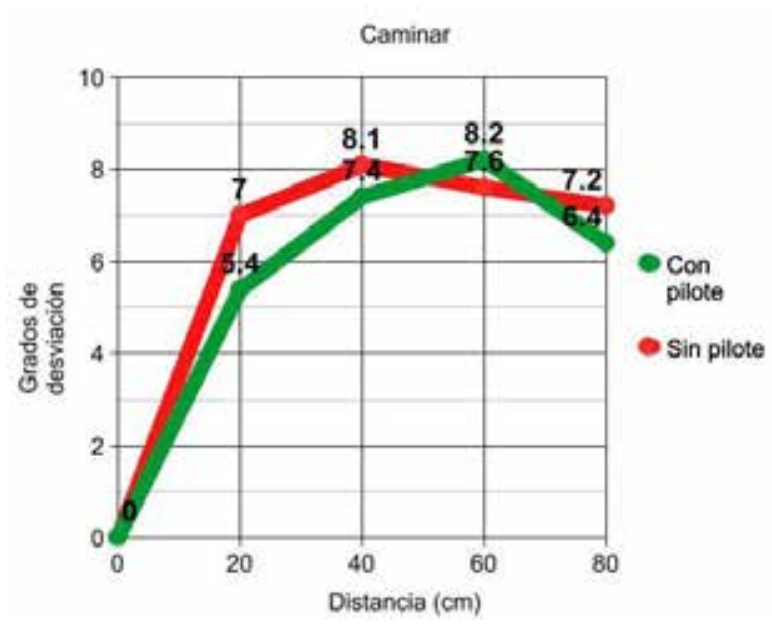

Figura 2. Resultados de desviación de la prueba caminar.

En cuanto a la situación de desbordamiento del río, el robot Bioloid constituye un buen prototipo para la solución del problema de desbordamiento, ya que por ser bípedo [16] [17] se desempeña mejor que un robot Rover [18] [19] [20] en las condiciones terrenales de las zonas de riberas, y así posteriormente implementar un plan macro para un prototipo más a la medida de la situación real.

\section{AGRADECIMIENTOS}

Los autores reconocen las contribuciones de la profesora Ingrid Durley Torres Pardo, quien dirigió el semillero de investigación Edubótica en la Funlam, así como la participación del profesor Mauricio López Bonilla del departamento de Ciencias Básicas en la Funlam. Su aporte fue fundamental en el apoyo al proceso de investigación mediante el cual se elaboró este artículo. Los autores también reconocen la colaboración del grupo de investigación SINTELWEB de la Universidad Nacional de Colombia, Facultad de Minas, quienes aportaron la infraestructura, el material técnico, colaboradores y los demás medios físicos que permitieron guiar el aprendizaje durante la realización de este trabajo.
TABLA 2

\begin{tabular}{cc} 
RESULTADOS DE VELOCIDAD CUANDO EL ROBOT GIRA \\
\hline Grados objetivo & Tiempo transcurrido \\
\hline $45^{\circ}$ & $14,63 \mathrm{~s}$ \\
$90^{\circ}$ & $26,43 \mathrm{~s}$ \\
$135^{\circ}$ & $37,61 \mathrm{~s}$ \\
$180^{\circ}$ & $49,51 \mathrm{~s}$ \\
\hline
\end{tabular}

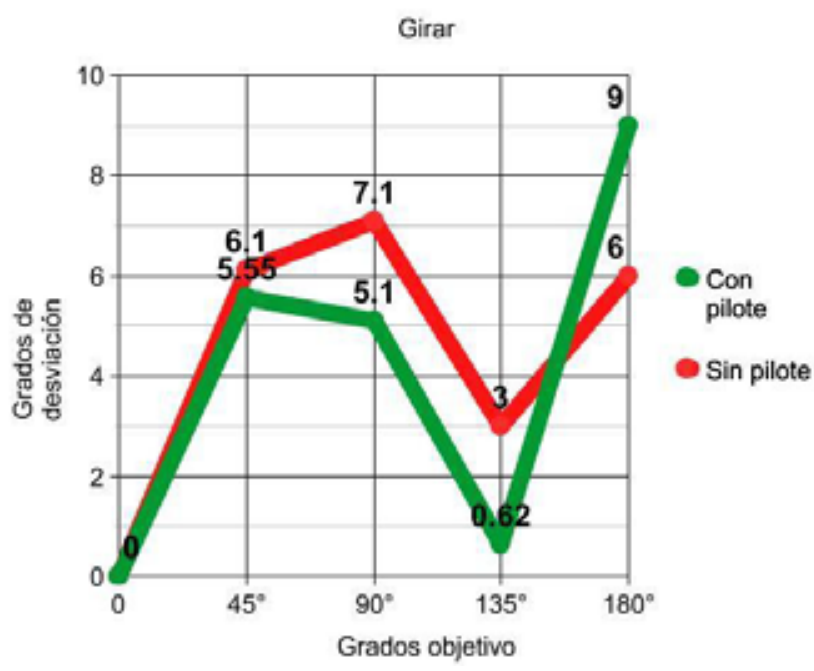

Figura 3. Resultados de desviación de la prueba girar.

\section{REFERENCIAS}

[1] F. M. Sánchez-Martín, S. P. Jiménez, R. F. Millán, J. Salvador-Bayarri, F. V. Monllau, R. J. Palou and M. H. Villavicencio. "Historia de la robótica: desde Arquitas de Tarento al Robot da Vinci". Institut de Robótica Industrial (IRI). Univ. Politécnica de Cataluña. CSIC. Madrid, España, pp. 185-196. Mar 2007.

[2] L. R. Batres Ayala and R. M. Guevara Vargas. "Investigación sobre los temas: Incertidumbre, Factores de la Incertidumbre, Razonamiento Aproximado y Razonamiento Inexacto". Universidad Don Bosco, pp. 1 - 16. Jun 30 de 2004.

[3] A. Betancurt, Robot para rescatar víctimas en desastres naturales [Online]. Colombia: Universidad Nacional de Colombia, Mar 2010 Disponible en: http://www.unperiodico.unal. edu.co/dper/article/robot-para-rescatar-victimas-en-desastres-naturales.html. 
[4] Paro-Robot, Therapeutic Robot [Online]. U.S. Disponible en: http://www.parorobots.com/

[5] S. Bermejo Sánchez. "Inteligencia y vida artificiales". In Desarrollo de robots basados en el comportamiento, J. G. Salgado. Edicions UPC: Barcelona, España, Sep 2003, pp. 2131.

[6] BajoEIAgua.com, Japón diseña un robot humanoide socorrista [Online]. BajoEIAgua. com, Sep 2012 Disponible en:

http://www.bajoelagua.com/mundo-submarino/noticias/2012-09-12/japon-disena-robothumanoide-socorrista-0854.html.

[7] Robotis, Robotis product Website [Online]. Japón: Robotis, 2012 Disponible en: http://www. robotis.com/xe/BIOLOID_main_en

[8] Robotis, Default Program (Bioloid Premium) [Online]. Japón: Robotis, 2012 Disponible en: http://support.robotis.com/en/product/bioloid/premiumkit/download/bioloid_premium_ demo.htm.

[9] Robotis, Gyro Sensor [Online]. Japón: Robotis, 2012 Disponible en: http://support.robotis. com/en/product/auxdevice/sensor/irsensor manual.htm.

[10] Conozcamos la Robótica, Robots según su arquitectura [Online]. Argentina: Conozcamos la Robótica, 2011 Disponible en: http:// conozcamoslarobotica.blogspot.com/p/robots-segun-su-arquitectura.html.

[11] Robotis, RoboPlus Task [Online]. Japón: Robotis, 2010 Disponible en: http://support. robotis.com/en/software/roboplus/roboplus_ task_main.htm

[12] Robotis, RoboPlus Motion [Online]. Japón: Robotis, 2010 Disponible en: http://support. robotis.com/en/software/roboplus/roboplus _ motion_main.htm
[13] A. Batalla Flores. "Habilidades Motrices", PI. Sant Pere. INDE Publicaciones: Barcelona, España, 2000, pp. 5-107.

[14] Bose Sanjay. "Hardware and software of personal computers", New Age International: New Delhi, U.S. 2007, pp. 1-224.

[15] B. C. Falgueras, "Qué es la Ingeniería del software". In Ingeniería del software, Diseñador: Manuel Andreu. Editorial UOC: Barcelona, España, Apr 2003, pp. 15-35.

[16] J. Larriva, E. Gillermo, J. Trelles and O. Vele. "Diseño Y Construcción De Un Robot Bípedo Experimental". In 2006 III Congreso Universidad y Cooperación al Desarrollo. Madrid, España, pp. 138-152.

[17] J. J. C. Martínez, E. T. Ortega, J. A. C. CastiIlo, J. L. G. Hernández, J. N. R. Castillo and M. C. C. A. Delgado, "Diseño y Construcción de un Robot Bípedo", Revista Internacional de educación en Ingeniería, vol. 1, pp. 1-14, 2008.

[18] N. Saeed. "Actuators and Drive Systems". In Introduction to Robotics, L. Ratts, VP \& Publisher: D. Fowley, Editorial Assistant: R. Manchiome, Marketing Manager: C. Ruel, Media Editor: L. Sapira, Production Manager: J. Soo, Assistant Production Editor: Y. L. Song. John Willey \& sons, Inc: U.S., 2011, pp. $266-319$.

[19] N. Sims, "Robot Rover", Baker \& Taylor: London, United Kingdom, Mar 2003, pp. 1-32.

[20] S. Dolphin, S. Dolphin Staff, N. Sims, "Robot Rover", San Val, Incorporated: London, United Kingdom, 2003 